\title{
Numerical Blow-up for A Heat Equation with Nonlinear Boundary Conditions
}

\author{
Kouamé Béranger Edja ${ }^{1}$, Kidjégbo Augustin Touré ${ }^{1}$ \& Brou Jean-Claude Koua ${ }^{2}$ \\ ${ }^{1}$ Institut National Polytechnique Houphouët-Boigny de Yamoussoukro, Côte d'Ivoire \\ ${ }^{2}$ Université Félix Houphouët-Boigny de Cocody, Côte d'Ivoire
}

Correspondence: Kouamé Béranger Edja, Laboratoire de Mathématiques et des Nouvelles Technologies de l'Information, Institut National Polytechnique Houphouët-Boigny de Yamoussoukro, Côte d'Ivoire.

Received: August 13, 2018 Accepted: August 28, 2018 Online Published: September 6, 2018

doi:10.5539/jmr.v10n5p119 URL: https://doi.org/10.5539/jmr.v10n5p119

\begin{abstract}
We study numerical approximations of solutions of a heat equation with nonlinear boundary conditions which produce blow-up of the solutions. By a semidiscretization using a finite difference scheme in the space variable we get a system of ordinary differential equations which is an approximation of the original problem. We obtain sufficient conditions which guarantee the blow-up solution of this system in a finite time. We also show that this blow-up time converges to the theoretical one when the mesh size goes to zero. We present some numerical results to illustrate certain point of our work.
\end{abstract}

Keywords: numerical blow-up, heat equation, nonlinear boundary, finite difference, arc length transformation, Aitken method

\section{Introduction}

In this paper, we study the behavior of a semidiscrete approximation of the following heat equation involving nonlinear boundary flux conditions :

$$
\left\{\begin{array}{l}
u_{t}=u_{x x}, \quad x \in(0,1), t \in(0, T) \\
u_{x}(0, t)=u^{p}(0, t), u_{x}(1, t)=u^{q}(1, t), t \in(0, T), \\
u(x, 0)=u_{0}(x), x \in[0,1]
\end{array}\right.
$$

where $p, q$ are positive constants. The initial function $u_{0}$ is a non-negative smooth function satisfying the compatibility conditions $u_{0}^{\prime}(0)=u_{0}^{p}(0), u_{0}^{\prime}(1)=u_{0}^{q}(1)$. Here $(0, T)$ is the maximum time interval on which the solution $u$ of $(1)$ exists. The time $T$ may be finite or infinite. When $T$ is infinite, we affirm that the solution $u$ exists globally. When $T$ is finite, the solution $u$ develops a singularity in a finite time, no matter how smooth $u_{0}$ is. Namely, we have

$$
\lim _{t \rightarrow T}\|u(\cdot, t)\|_{\infty}=+\infty
$$

where $\|u(\cdot, t)\|_{\infty}=\max _{0 \leq x \leq 1}|u(x, t)|$. In this previous case, we affirm that the solution $u$ blows up in a finite time and this time is called the blow-up time of the solution $u$.

The theoretical study of blow-up of solutions for heat equations with nonlinear boundary conditions has been the subject of investigations of many authors (Gomez, Márquez \& Wolanski, 1991; Hu \& Yin, 1994; Levine \& Payne, 1974; Ozalp \& Selcusk, 2015; Wang \& Wu, 2001; Yang \& Zhou, 2016; and the references cited therein). In (Ozalp \& Selcusk, 2015) Ozalp and Selcuk show that under certain conditions, any positive solution of the problem (1) must blow up in a finite time and the blow-up point occurs only at the boundary $x=1$. In this paper, we are interested in the numerical study using a semidiscrete scheme of (1). For previous study on numerical approximations of parabolic system with non-linear boundary conditions we refer to (Abia, Lopez-Marcos \& Martinez, 1996; Taha, Toure \& Mensah, 2012; Toure, N'Guessan \& Diabate, 2015; Ushijima, 2000). In (Ushijima, 2000) Ushijima presents rather simple but general sufficient conditions which guarantee that the blow-up time for the original equation is well approximated by that for approximate equations. By using a theorem of Ushijima (Ushijima, 2000) and under certain conditions we show that any positive solution of semidiscrete scheme of (1) blows up in a finite time and the semidiscrete blow-up time converges to the theoretical one when the mesh size goes to zero.

The rest of the paper is organized as follows : in the next section, we present a semidiscrete scheme of the problem (1). In Section 3, we give some properties concerning our semidiscrete scheme. In Section 4, under some conditions, we prove that the solution of the semidiscrete scheme of (1) blows up in a finite time and this blow-up time converges to the theoretical one when the mesh size goes to zero. Finally, in the last section, we give some numerical results to illustrate our analysis. 


\section{The Semidiscrete Problem}

We introduce the following uniform mesh on the interval $[0,1]$

$$
x_{i}=(i-1) h, i=1, \ldots, I, h=1 /(I-1) .
$$

We denote $U_{h}=U_{h}(t)=\left(U_{1}(t), \ldots, U_{I}(t)\right)^{T}$ the values of the numerical approximation at the nodes $x_{i}$ at time $t$. For semidiscretization of problem (1) we use a finite difference method and obtain the following system of ODEs :

$$
\begin{gathered}
\frac{d U_{i}(t)}{d t}=\frac{U_{i-1}(t)-2 U_{i}(t)+U_{i+1}(t)}{h^{2}}, i=2, \ldots, I-1, t>0, \\
\frac{d U_{1}(t)}{d t}=\frac{2 U_{2}(t)-2 U_{1}(t)}{h^{2}}-\frac{2 U_{1}^{p}(t)}{h}, t>0, \\
\frac{d U_{I}(t)}{d t}=\frac{2 U_{I-1}(t)-2 U_{I}(t)}{h^{2}}+\frac{2 U_{I}^{q}(t)}{h}, t>0, \\
U_{i}(0)=\varphi_{i} \geq 0, \quad i=1, \ldots, I,
\end{gathered}
$$

where $\varphi_{i+1} \geq \varphi_{i}, 1 \leq i \leq I$ and

$$
\begin{gathered}
\delta^{2} U_{i}(t)=\frac{U_{i-1}(t)-2 U_{i}(t)+U_{i+1}(t)}{h^{2}}, 2 \leq i \leq I-1, t \in\left(0, T_{h}\right), \\
\delta^{2} U_{1}(t)=\frac{2 U_{2}(t)-2 U_{1}(t)}{h^{2}}-\frac{2 U_{1}^{p}(t)}{h}, t \in\left(0, T_{h}\right), \\
\delta^{2} U_{I}(t)=\frac{2 U_{I-1}(t)-2 U_{I}(t)}{h^{2}}+\frac{2 U_{I}^{q}(t)}{h}, t \in\left(0, T_{h}\right) .
\end{gathered}
$$

Here $\left(0, T_{h}\right)$ is the maximum time interval on which $\left\|U_{h}(t)\right\|_{\infty}$ is finite. When $T_{h}$ is finite, we affirm that the solution $U_{h}$ blows up in a finite time and the time $T_{h}$ is called the blow-up time of the solution $U_{h}$.

\section{Properties of the Semidiscrete Scheme}

In this section, we give some auxiliary results for the problem (1).

Definition 1 A function $V_{h} \in C^{1}\left(\left(0, T_{h}\right), \mathbb{R}^{I}\right)$ is a lower solution of (2)-(5) if

$$
\begin{gathered}
\frac{d V_{i}(t)}{d t}-\frac{V_{i-1}(t)-2 V_{i}(t)+V_{i+1}(t)}{h^{2}} \leq 0, i=2, \ldots, I-1, t>0, \\
\frac{d V_{1}(t)}{d t}-\frac{2 V_{2}(t)-2 V_{1}(t)}{h^{2}}+\frac{2 V_{1}^{p}(t)}{h} \leq 0, t>0, \\
\frac{d V_{I}(t)}{d t}-\frac{2 V_{I-1}(t)-2 V_{I}(t)}{h^{2}}-\frac{2 V_{I}^{q}(t)}{h} \leq 0, t>0, \\
V_{i}(0) \leq U_{i}(0), i=1, \ldots, I,
\end{gathered}
$$

where $U_{h}=\left(U_{1}, \ldots, U_{I}\right)^{T}$ is solution of (2)-(5). On the other hand, we say that $V_{h} \in C^{1}\left(\left(0, T_{h}\right), \mathbb{R}^{I}\right)$ is an upper solution of (2)-(5) if these inequalities are reversed.

lemma 2 Let $W_{h}, V_{h} \in C^{1}\left(\left(0, T_{h}\right), \mathbb{R}^{I}\right)$ be lower and upper solutions of (2)-(5) respectively, then

$$
W_{h}(t) \leq V_{h}(t), \forall t \in\left(0, T_{h}\right) .
$$

Proof Let us define the vector $Z_{h}(t)=\left(V_{h}(t)-W_{h}(t)\right) e^{\lambda t}$ with $\lambda$ a real. We have

$$
\begin{gathered}
\frac{d Z_{i}(t)}{d t}-\frac{Z_{i-1}(t)-2 Z_{i}(t)+Z_{i+1}(t)}{h^{2}}-\lambda Z_{i}(t) \geq 0, \quad i=2, \ldots, I-1, t \in\left(0, T_{h}\right), \\
\frac{d Z_{1}(t)}{d t}-\frac{2 Z_{2}(t)-2 Z_{1}(t)}{h^{2}}+\left(-\lambda+\frac{2 p \zeta^{p-1}(t)}{h}\right) Z_{1}(t) \geq 0, t \in\left(0, T_{h}\right),
\end{gathered}
$$




$$
\begin{gathered}
\frac{d Z_{I}(t)}{d t}-\frac{2 Z_{I-1}(t)-2 Z_{I}(t)}{h^{2}}+\left(-\lambda-\frac{2 q \xi^{q-1}(t)}{h}\right) Z_{I}(t) \geq 0, t \in\left(0, T_{h}\right), \\
Z_{i}(0) \geq 0, \quad i=1, \ldots, I,
\end{gathered}
$$

where $\zeta(t), \xi(t)$ lie, respectively, between $V_{1}(t)$ and $W_{1}(t)$, and between $V_{I}(t)$ and $W_{I}(t)$.

Denote $m=\min _{1 \leq i \leq I, t \in\left[0, T_{0}\right]} Z_{i}(t)$ where $T_{0} \in\left(0, T_{h}\right)$. Since for $i \in\{1, \ldots, I\}, Z_{i}(t)$ is a continuous function in the compact $\left[0, T_{0}\right]$, there exists $i_{0} \in\{1, \ldots, I\}$ such that $m=Z_{i_{0}}\left(t_{i_{0}}\right)$. Assume $m<0$.

Taking $\lambda$ negative such that

$$
\frac{2 p \zeta^{p-1}\left(t_{i_{0}}\right)}{h}-\lambda>0 \text { and }-\frac{2 q \xi^{q-1}\left(t_{i_{0}}\right)}{h}-\lambda>0
$$

If $t_{i_{0}}=0$, then $Z_{i_{0}}(0)<0$, which contradicts (9), hence $t_{i_{0}} \neq 0$;

if $1 \leq i_{0} \leq I$, we have

$$
\begin{gathered}
\frac{d Z_{i_{0}}\left(t_{i_{0}}\right)}{d t}=\lim _{k \rightarrow 0} \frac{Z_{i_{0}}\left(t_{i_{0}}\right)-Z_{i_{0}}\left(t_{i_{0}}-k\right)}{k} \leq 0, \\
\frac{Z_{i_{0}-1}\left(t_{i_{0}}\right)-2 Z_{i_{0}}\left(t_{i_{0}}\right)+Z_{i_{0}+1}\left(t_{i_{0}}\right)}{h^{2}} \geq 0 \text { if } 2 \leq i_{0} \leq I-1, \\
\frac{2 Z_{i_{0}+1}\left(t_{i_{0}}\right)-2 Z_{i_{0}}\left(t_{i_{0}}\right)}{h^{2}} \geq 0 \text { if } i_{0}=1, \\
\frac{2 Z_{i_{0}-1}\left(t_{i_{0}}\right)-2 Z_{i_{0}}\left(t_{i_{0}}\right)}{h^{2}} \geq 0 \text { if } i_{0}=I .
\end{gathered}
$$

Moreover by a straightforward computation we get

$$
\begin{aligned}
& \frac{d Z_{i_{0}}\left(t_{i_{0}}\right)}{d t}-\frac{Z_{i_{0}-1}\left(t_{i_{0}}\right)-2 Z_{i_{0}}\left(t_{i_{0}}\right)+Z_{i_{0}+1}\left(t_{i_{0}}\right)}{h^{2}}-\lambda Z_{i_{0}}\left(t_{i_{0}}\right)<0, \\
& \frac{d Z_{1}\left(t_{i_{0}}\right)}{d t}-\frac{2 Z_{2}\left(t_{i_{0}}\right)-2 Z_{1}\left(t_{i_{0}}\right)}{h^{2}}+\left(\frac{2 p \zeta^{p-1}\left(t_{i_{0}}\right)}{h}-\lambda\right) Z_{1}\left(t_{i_{0}}\right)<0, \\
& \frac{d Z_{I-1}\left(t_{i_{0}}\right)}{d t}-\frac{2 Z_{I-1}\left(t_{i_{0}}\right)-2 Z_{I}\left(t_{i_{0}}\right)}{h^{2}}+\left(-\frac{2 q \xi^{q-1}\left(t_{i_{0}}\right)}{h}-\lambda\right) Z_{I}\left(t_{i_{0}}\right)<0,
\end{aligned}
$$

but these inequalities contradict (6)-(8) and the proof is complete.

The results of the next lemma are analogue to those of continuous problem.

lemma 3 Let $U_{h} \in C^{1}\left(\left(0, T_{h}\right), \mathbb{R}^{I}\right)$ be solution of (2)-(5) with $\varphi_{i} \geq 0$ such that $\delta^{2} \varphi_{i} \geq 0$ and $\varphi_{i+1}>\varphi_{i}$, for $i=1, \ldots, I-1$. Then we have

(i) $U_{i}(t) \geq 0$ and $U_{i}(t) \geq \varphi_{i}, \quad i=1, \ldots, I, \quad t \in\left(0, T_{h}\right)$;

(ii) $U_{i+1}(t)>U_{i}(t), \quad i=1, \ldots, I-1, \quad t \in\left(0, T_{h}\right)$;

(iii) $\frac{d U_{i}(t)}{d t}>0, \quad i=1, \ldots, I, t \in\left(0, T_{h}\right)$.

Proof

(i) $\frac{d}{d t} \varphi_{i}-\delta^{2} \varphi_{i} \leq 0, i=1, \ldots, I$, hence $\varphi_{h}$ is a lower solution of (2)-(5). According to the lemma 2 we have

$$
U_{i}(t) \geq \varphi_{i} \geq 0, \quad \text { for } \quad i=1, \ldots, I, t \in\left(0, T_{h}\right) .
$$


(ii) Let $t_{0}$ be the first $t>0$, such that $K_{i}(t)=U_{i+1}(t)-U_{i}(t)>0$, for $1 \leq i \leq I-1$, but $K_{i_{0}}\left(t_{0}\right)=U_{i_{0}+1}\left(t_{0}\right)-U_{i_{0}}\left(t_{0}\right)=0$ for a certain $i_{0} \in\{1, \ldots, I-1\}$. Without lost of generality, we can suppose that $i_{0}$ is the smallest integer which satisfies the above equality. We have

$$
\begin{gathered}
\frac{d K_{i_{0}}\left(t_{0}\right)}{d t}=\lim _{\epsilon \rightarrow 0} \frac{K_{i_{0}}\left(t_{0}\right)-K_{i_{0}}\left(t_{0}-\epsilon\right)}{\epsilon} \leq 0, \\
\frac{K_{i_{0}+1}\left(t_{0}\right)-2 K_{i_{0}}\left(t_{0}\right)+K_{i_{0}-1}\left(t_{0}\right)}{h^{2}}>0 \quad \text { if } 2 \leq i_{0} \leq I-2, \\
\frac{K_{i_{0}+1}\left(t_{0}\right)-3 K_{i_{0}}\left(t_{0}\right)}{h^{2}}>0 \quad \text { if } i_{0}=1, \\
\frac{-3 K_{i_{0}}\left(t_{0}\right)+K_{i_{0}-1}\left(t_{0}\right)}{h^{2}}>0 \quad \text { if } i_{0}=I-1,
\end{gathered}
$$

which implies that

$$
\begin{gathered}
\frac{d K_{i_{0}}\left(t_{0}\right)}{d t}-\frac{K_{i_{0}+1}\left(t_{0}\right)-2 K_{i_{0}}\left(t_{0}\right)+K_{i_{0}-1}\left(t_{0}\right)}{h^{2}}<0 \quad \text { if } 2 \leq i_{0} \leq I-2, \\
\frac{d K_{i_{0}}\left(t_{0}\right)}{d t}-\frac{K_{i_{0}+1}\left(t_{0}\right)-3 K_{i_{0}}\left(t_{0}\right)}{h^{2}}-\frac{2 U_{i_{0}}^{p}(t)}{h}<0 \quad \text { if } i_{0}=1, \\
\frac{d K_{i_{0}}\left(t_{0}\right)}{d t}-\frac{-3 K_{i_{0}}\left(t_{0}\right)+K_{i_{0}-1}\left(t_{0}\right)}{h^{2}}-\frac{2 U_{i_{0}+1}^{q}(t)}{h}<0 \quad \text { if } i_{0}=I-1,
\end{gathered}
$$

but these contradict (2)-(4) and we obtain the desired result.

(iii) Denote $Z_{i}(t)=U_{i}(t+\varepsilon)-U_{i}(t), \quad i=1, \ldots, I$, using (i) we obtain $Z_{i}(0) \geq 0$.

A straightforward calculation yields

$$
\begin{gathered}
\frac{d Z_{i}(t)}{d t}=\frac{Z_{i-1}(t)-2 Z_{i}(t)+Z_{i+1}(t)}{h^{2}}, 2 \leq i \leq I-1, t \in\left(0, T_{h}\right), \\
\frac{d Z_{1}(t)}{d t}=\frac{2 Z_{2}(t)-2 Z_{1}(t)}{h^{2}}-\frac{2 p \eta^{p-1}(t) Z_{1}(t)}{h}, t \in\left(0, T_{h}\right), \\
\frac{d Z_{I}(t)}{d t}=\frac{2 Z_{I-1}(t)-2 Z_{I}(t)}{h^{2}}+\frac{2 q \xi^{q-1}(t) Z_{I}(t)}{h}, t \in\left(0, T_{h}\right), \\
Z_{i}(0) \geq 0, \quad i=1, \ldots, I,
\end{gathered}
$$

where $\eta(t), \xi(t)$ lie, respectively, between $U_{1}(t+\varepsilon)$ and $U_{1}(t)$ and between $U_{I}(t+\varepsilon)$ and $U_{I}(t)$. Below inequality can easily be proved in a manner similar to that of lemma 2

$$
Z_{i}(t) \geq 0, \quad i=1, \ldots, I, \forall t \in\left(0, T_{h}\right) .
$$

This fact implies the desired result.

The next theorem establishes that for each fixed time interval $\left[0, T^{*}\right]$ where the solution $u$ of (1) is defined, the solution of the semidiscrete problem (2)-(5) approximates $u$, as $h \rightarrow 0$. This theorem will be used in the study of the convergence of the blow-up time of the semidiscrete problem.

Theorem 4 Assume that the problem (1) has a solution $u \in C^{4,1}\left([0,1] \times\left[0, T^{*}\right]\right)$ and the initial condition $\varphi_{h}$ at (5) verifies

$$
\left\|\varphi_{h}-u_{h}(0)\right\|_{\infty}=o(1), h \rightarrow 0,
$$

where $u_{h}(t)=\left(u\left(x_{1}, t\right), \ldots, u\left(x_{I}, t\right)\right)^{T}$. Then, for $h$ small enough, the semidiscrete problem (2)-(5) has a unique solution $U_{h} \in C^{1}\left(\left[0, T^{*}\right], \mathbb{R}^{I}\right)$ such that

$$
\max _{t \in\left[0, T^{*}\right]}\left\|U_{h}(t)-u_{h}(t)\right\|_{\infty}=O\left(\left\|\varphi_{h}-u_{h}(0)\right\|_{\infty}+h^{2}\right) \text {, as } h \rightarrow 0 .
$$

Proof. Let $\sigma>0$ be such that

$$
\|u\|_{\infty}<\sigma, t \in\left[0, T^{*}\right]
$$


Then the problem (2)-(5) has for each $h$, a unique solution $U_{h} \in C^{1}\left(\left[0, T^{*}\right], \mathbb{R}^{I}\right)$. Let $t(h) \leq T^{*}$ be the greatest value of $t>0$ such that

$$
\left\|U_{h}(t)-u_{h}(t)\right\|_{\infty}<1 .
$$

The relation (10) implies $t(h)>0$ for $h$ small enough. Using the triangle inequality, we obtain

$$
\left\|U_{h}(t)\right\|_{\infty} \leq\left\|U_{h}(t)-u_{h}(t)\right\|_{\infty}+\|u(\cdot, t)\|_{\infty}, \text { for } t \in(0, t(h)),
$$

which implies that

$$
\left\|U_{h}(t)\right\|_{\infty} \leq 1+\sigma, \text { for } t \in(0, t(h)) .
$$

Let $e_{h}(t)=U_{h}(t)-u_{h}(t), \forall t \in\left[0, T^{*}\right]$ be the discretization error and let $W_{h} \in C^{1}\left(\left[0, T^{*}\right], \mathbb{R}^{I}\right)$ be such that $W_{i}(t)=\left(\left\|\varphi_{h}-u_{h}(0)\right\|_{\infty}+M h^{2}\right) e^{(M+1) t}, i=1, \ldots, I, \forall t \in\left[0, T^{*}\right]$, with $M$ a positive constant. We can prove by the lemma 2 that

$$
\left|e_{i}(t)\right|<W_{i}(t), 1 \leq i \leq I, \text { for } t \in(0, t(h)) .
$$

We deduce that

$$
\left\|U_{h}(t)-u_{h}(t)\right\|_{\infty} \leq\left(\left\|\varphi_{h}-u_{h}(0)\right\|_{\infty}+M h^{2}\right) e^{(M+1) t}, \text { for } t \in(0, t(h)) .
$$

Suppose that $T^{*}>t(h)$ from 12 , we obtain

$$
1=\left\|U_{h}(t(h))-u_{h}(t(h))\right\|_{\infty} \leq\left(\left\|\varphi_{h}-u_{h}(0)\right\|_{\infty}+M h^{2}\right) e^{(M+1) t} .
$$

Since the term on the right hand side of the above inequality goes to zero as $\mathrm{h}$ tends to zero, we deduce that, $1 \leq 0$, which is impossible. Hence we have $t(h)=T^{*}$, and the proof is complete.

\section{Numerical Blow-Up}

In this section, under some assumptions, we show that for each solution of (1) with a nonnegative initial data, there exists a unique solution of the semidiscrete problem (2)-(5) which blows up in finite time and this time converges to the real one when the mesh size goes to zero. For this fact, we show that the hypothesis of the Theorem 1.4 in (Ushijima, 2000) are satisfied.

Step 1 (blow-up of $U_{h}$ ). Suppose that there exists a unique local solution $u$ of problem (1) which blow up in finite time $T$ for an initial data $u_{0}$. Assume that the hypothesis of the Theorem 4 hold.

Then, for $h$ small enough, the semidiscrete problem (2)-(5) has a unique solution $U_{h} \in C^{1}\left(\left[0, T^{*}\right], \mathbb{R}^{I}\right)$ with $T^{*}<T$. For the following we define the energy $I$ by

$$
I[u](t)=\frac{1}{2} \int_{0}^{1}\left|u_{x}\right|^{2} d x-\frac{1}{q+1} u^{q+1}(1, t)+\frac{1}{p+1} u^{p+1}(0, t), \forall t \in[0, T) .
$$

The derivative $I[u](t)$ is given by

$$
\frac{d I[u](t)}{d t}=-\int_{0}^{1}\left(u_{t}(x, t)\right)^{2} d x<0, \forall t \in[0, T) .
$$

Introduce a function $J$ as follows

$$
J(t)=\int_{0}^{1}(u(x, t))^{2} d x, \forall t \in[0, T) .
$$

Then we have

$$
\frac{d J(t)}{d t}=-4 I[u](t)+\frac{2(q-1)}{q+1} u^{q+1}(1, t)-\frac{2(p-1)}{p+1} u^{p+1}(0, t), \quad \forall t \in[0, T) .
$$

As a consequence of (14) we obtain the following inequality

$$
\frac{d J(t)}{d t} \geq-4 I\left[u_{0}\right]+\frac{2(q-1)}{q+1} u^{q+1}(1, t)-\frac{2(p-1)}{p+1} u^{p+1}(0, t), \forall t \in[0, T) .
$$

Set $H(t)=-4 I\left[u_{0}\right]+\frac{2(q-1)}{q+1} u^{q+1}(1, t)-\gamma, \forall t \in[0, T)$, where

$\gamma=\sup \left\{\frac{2(p-1)}{p+1} u^{p+1}(0, t): t \in[0, T]\right\}$, since $u(0, \cdot)$ is continuous function in $[0, T]$. We deduce that $\frac{d J(t)}{d t} \geq H(t)$, 
$\forall t \in[0, T)$, and $\lim _{t \rightarrow T} H(t)=\infty$ since the blow-up point of $u$ is $x=1$.

Hence the condition $\left(b^{\prime}\right)$ of (Ushijima, 2000) is checked. Now we denote by

$$
\begin{gathered}
I_{h}\left[U_{h}\right](t)=h \sum_{i=1}^{I-1}\left(\frac{1}{h}\left(U_{i+1}(t)-U_{i}(t)\right)\right)^{2}+\frac{1}{p+1} U_{1}^{p+1}(t)-\frac{1}{q+1} U_{I}^{q+1}(t), \\
J_{h}(t)=h \sum_{i=1}^{I} U_{i}^{2}(t),
\end{gathered}
$$

and

$$
H_{h}(t)=-I_{h}\left[U_{h}\right](0)+\frac{2(q-1)}{q+1} U_{I}^{q+1}(t)-\gamma
$$

numerical approximations of $I, J$ and $H$, respectively.

By the Theorem 4 it is easy to see that the condition $\left(A 2^{\prime}\right)$ of (Ushijima, 2000) holds. Namely, for any $\varepsilon>0$,

$$
\lim _{h \rightarrow 0} \sup _{t \in[0, T-\varepsilon]}\left|J(t)-J_{h}(t)\right|=0 \quad \text { and } \quad \lim _{h \rightarrow 0} \sup _{t \in[0, T-\varepsilon]}\left|H(t)-H_{h}(t)\right|=0 .
$$

Remark that

$$
\frac{2(q-1)}{q+1} u^{q+1}(1, t)=2(q-1) \int_{0}^{1} u^{q}(x, t) u_{x}(x, t) d x+\frac{2(q-1)}{q+1} u^{q+1}(0, t) .
$$

According to (16), we obtain

$$
\frac{d J(t)}{d t} \geq-4 I\left[u_{0}\right]+2(q-1) \int_{0}^{1}(u(x, t))^{q} u_{x}(x, t) d x+\frac{2(q-1)}{q+1} u^{q+1}(0, t)-\frac{2(p-1)}{p+1} u^{p+1}(0, t) .
$$

When we assume that the initial data satisfies,

$$
u_{x x}(x, 0) \geq 0,
$$

we have $u_{t}>0$ in $(0,1) \times(0, T)$ (see Ozalp, \& Selcusk, 2015), which implies that $u_{x}$ is monotone increasing. Thus, we obtain

$$
\frac{d J(t)}{d t} \geq-4 I\left[u_{0}\right]+2(q-1) u_{x}(0, t) \int_{0}^{1}(u(x, t))^{q} d x+\frac{2(q-1)}{q+1} u^{q+1}(0, t)-\frac{2(p-1)}{p+1} u^{p+1}(0, t),
$$

hence

$$
\frac{d J(t)}{d t} \geq \beta \int_{0}^{1}(u(x, t))^{q} d x+\alpha+\Gamma,
$$

where $\alpha=\min \left\{-4 I\left[u_{0}\right],-4 I_{h}\left[\varphi_{h}\right]\right\}, \Gamma=\min \left\{\Gamma_{1}, \Gamma_{2}\right\}, \beta=2(q-1) \min \left\{u_{x}(0, t): t \in[0, T]\right\}$, with

$$
\begin{aligned}
& \Gamma_{1}=\inf \left\{\frac{2(q-1)}{q+1} u^{q+1}(0, t)-\frac{2(p-1)}{p+1} u^{p+1}(0, t): t \in[0, T]\right\}, \\
& \Gamma_{2}=\inf \left\{\frac{2(q-1)}{q+1} U_{1}^{q+1}(t)-\frac{2(p-1)}{p+1} U_{1}^{p+1}(0, t): t \in[0, T]\right\},
\end{aligned}
$$

and

$$
I_{h}\left[\varphi_{h}\right]=h \sum_{i=1}^{I-1}\left(\frac{1}{h}\left(U_{i+1}(0)-U_{i}(0)\right)\right)^{2}+\frac{1}{p+1} U_{1}^{p+1}(0)-\frac{1}{q+1} U_{I}^{q+1}(0) .
$$

By Jensen's inequality, we have

$$
\frac{d J(t)}{d t} \geq \beta(J)^{\frac{q}{2}}+\alpha+\Gamma
$$


with $q>2$.

Let $G(s)=\beta(s)^{\frac{q}{2}}+\alpha+\Gamma$.

Therefore it exists $R$ such that

$$
\left\{\begin{array}{l}
G(s)>0, \quad \forall s>R \\
\int_{R}^{\infty} \frac{d s}{G(s)}<+\infty, \quad \forall q>2 .
\end{array}\right.
$$

A straightforward calculation yields us the following inequality

$$
\frac{d J_{h}(t)}{d t} \geq G\left(J_{h}\right)
$$

We can see that $J_{h}$ is a $C^{1}$ function satisfying the inequality (I1) of (Ushijima, 2000).

The hypothesis $\left(b^{\prime}\right)$, (I1) and ( $\left.A 2^{\prime}\right)$ guarantee the blow-up of the solution $U_{h}$ of (2)-(5) in finite time $T_{h}$.

Step 2 (Convergence of blow-up time). From (17) we have

$$
\frac{d I_{h}\left[U_{h}\right](t)}{d t}=-\frac{h}{2}\left(U_{1}^{\prime}(t)\right)^{2}-h \sum_{i=2}^{I-1}\left(U_{i}^{\prime}(t)\right)^{2}-\frac{h}{2}\left(U_{I}^{\prime}(t)\right)^{2} \leq 0,
$$

and

$$
\frac{d H_{h}(t)}{d t}=2(q-1) U_{I}^{q}(t) \frac{d U_{I}(t)}{d t} .
$$

A simple computation gives

$$
\begin{gathered}
\frac{d J_{h}(t)}{d t} \geq H_{h}(t) . \\
\frac{d H_{h}(t)}{d t} \geq 0, \text { according to Lemma } 3 .
\end{gathered}
$$

It is easy to see that the hypothesis (I1') in (Ushijima, 2000) is satisfies.

By virtue of Theorem 1.4 in (Ushijima, 2000), the results are obtained as desired.

\section{Numerical Simulation}

In this section, we present some numerical approximations to the blow-up time of (2)-(5) for the initial data $\varphi_{i}=(i-1) h$, $i=1, \ldots, I$, lower solution of (2)-(5) with different values of $q$ and $p$, where $q \geq 3$ and $q \geq p$. Here the numerical results are given by the algorithm proposed by C. Hirota and K. Ozawa (Hirota \& Ozawa, 2006), we briefly present this algorithm. Consider the system of ODEs (2)-(5). We consider the variables $t$ and $U_{i}$ as functions of the arc length $s$. Since $d s^{2}=d t^{2}+d U_{1}^{2}+\cdots+d U_{I}^{2}$, the variables $t(s)$ and $U_{i}(s)$ satisfy the system of differential equations

$$
\left\{\begin{array}{l}
\frac{d t}{d s}=\frac{1}{\sqrt{1+\sum_{i=1}^{I} f_{i}^{2}}}, \\
\frac{d U_{i}}{d s}=\frac{f_{i}}{\sqrt{1+\sum_{i=1}^{I} f_{i}^{2}}}, i=1, \ldots, I, \\
t(0)=0, U_{i}(0)=\varphi_{i} \geq 0, \quad i=1, \ldots, I,
\end{array}\right.
$$

where $0<s<\infty$, and

$$
\left(\begin{array}{c}
f_{1} \\
\vdots \\
\vdots \\
\vdots \\
f_{I}
\end{array}\right)=\frac{1}{h^{2}}\left(\begin{array}{ccccc}
-2 & 2 & 0 & \ldots & 0 \\
1 & -2 & 1 & \ddots & \vdots \\
0 & \ddots & \ddots & \ddots & 0 \\
\vdots & \ddots & 1 & -2 & 1 \\
0 & \ldots & 0 & 2 & -2
\end{array}\right)\left(\begin{array}{c}
U_{1} \\
\vdots \\
\vdots \\
\vdots \\
U_{I}
\end{array}\right)+\left(\begin{array}{c}
-\frac{2 U_{1}^{p}}{h} \\
0 \\
\vdots \\
0 \\
\frac{2 U_{I}^{q}}{h}
\end{array}\right)
$$

It is well known (Hirota \& Ozawa, 2006) that 


$$
\lim _{s \rightarrow \infty} t(s)=T_{h}, \text { and } \lim _{s \rightarrow \infty}\left\|U_{h}(s)\right\|_{\infty}=\infty .
$$

For the numerical computation, let us define $s=s_{l}$ by $s_{l}=2^{16} \cdot 2^{l}(l=0,1, \ldots, 12)$. For each value of $l$, we apply DOP54 (see Hairer, Nørsett \& Wanner, 1993) to system (19) and we get a linearly convergent sequence to the blow-up time $\left\{t_{l}^{(k)}\right\}_{k=1}^{l+1}$. We also accelerate the sequence recursively by Aitken method's :

$$
t_{l+2}^{(k+1)}=t_{l+1}^{(k)}-\frac{\left(t_{l+2}^{(k)}-t_{l+1}^{(k)}\right)^{2}}{t_{l+2}^{(k)}-2 t_{l+1}^{(k)}+t_{l}^{(k)}}, \quad l \geq 2 k, k=0,1,2, \ldots
$$

As in (Hirota \& Ozawa, 2006), for our experiments we set RTOL $=$ ATOL $=1 . d-15$ and ITOL $=0$. Where the parameters RTOL and ATOL are the tolerances of the relative and absolute errors, respectively, and ITOL is used to choose the manner in which the errors are controlled.

Tables and graphics : $\varphi_{i}=(i-1) h, i=1, \cdots, I$

In the following tables, in rows, we present the numerical blow-up times $T_{h}$, the numbers of iterations $n$, orders of the approximations $s$ corresponding to meshes of $16,32,64,128,256,512,1024$. Approximation orders are calculated by

$$
s=\frac{\log \left(\left(T_{4 h}-T_{2 h}\right) /\left(T_{2 h}-T_{h}\right)\right)}{\log (2)}, \text { where } h=1 /(I-1) .
$$

Remark 5. The various tables of our numerical results show that there is a relationship between the blow-up time and the flow on the boundary. If we consider the problem (2)-(5) in the case where the initial data $\varphi(x)=x$ and $q=3$, we observe from tables 1-3 that the numerical blow-up time is approximately equal to 0.3. When $q=4$, we observe from Tables 4-7 that the numerical blow-up time is approximately equal to 0.2. Thus we can said that when rise $q$ we have an acceleration of blow-up of the solution. On the other hand, when $q$ is fixed and $p$ is increased, we observe from tables 1-3 and 4-7 that there is a slight diminishes of the blow-up time. Also, from the tables we observe the convergence of blow-up time $T_{h}$ of the solution of (2)-(5), since the rate of convergence is near 2. This result does not surprise us because of the result established in the previous section. 


\begin{tabular}{|c|c|c|c|}
\hline$I$ & $T_{h}$ & $n$ & $\mathrm{~s}$ \\
\hline 16 & 0.264970 & 12527 & - \\
32 & 0.260386 & 15008 & - \\
64 & 0.259024 & 19880 & 1.75 \\
128 & 0.258625 & 31186 & 1.77 \\
256 & 0.258510 & 53933 & 1.79 \\
512 & 0.258477 & 114522 & 1.80 \\
1024 & 0.258468 & 357602 & 1.87 \\
\hline
\end{tabular}

Table 1. Numerical blow-up times, number of iterations and order of the approximations obtained for $q=3$, $p=3$.

\begin{tabular}{|c|c|c|c|}
\hline$I$ & $T_{h}$ & $n$ & $\mathrm{~s}$ \\
\hline 16 & 0.277556 & 12317 & - \\
32 & 0.272857 & 14343 & - \\
64 & 0.271467 & 18685 & 1.76 \\
128 & 0.271061 & 28043 & 1.77 \\
256 & 0.270944 & 50319 & 1.79 \\
512 & 0.270911 & 117269 & 1.82 \\
1024 & 0.270902 & 373244 & 1.87 \\
\hline
\end{tabular}

Table 3. Numerical blow-up times, number of iterations and order of the approximations obtained for $q=3$, $p=1$.

\begin{tabular}{|c|c|c|c|}
\hline$I$ & $T_{h}$ & $n$ & $\mathrm{~s}$ \\
\hline 16 & 0.193469 & 8670 & - \\
32 & 0.189455 & 10563 & - \\
64 & 0.188243 & 14549 & 1.73 \\
128 & 0.187885 & 22704 & 1.76 \\
256 & 0.187781 & 39660 & 1.78 \\
512 & 0.187752 & 84020 & 1.84 \\
1024 & 0.187744 & 260848 & 1.86 \\
\hline
\end{tabular}

Table 5. Numerical blow-up times, number of iterations and order of the approximations obtained for $q=4$, $p=3$.

\begin{tabular}{|c|c|c|c|}
\hline$I$ & $T_{h}$ & $n$ & $\mathrm{~s}$ \\
\hline 16 & 0.199975 & 8499 & - \\
32 & 0.195868 & 9986 & - \\
64 & 0.194633 & 13108 & 1.73 \\
128 & 0.194270 & 19838 & 1.77 \\
256 & 0.194165 & 36135 & 1.79 \\
512 & 0.194135 & 84981 & 1.81 \\
1024 & 0.194128 & 268863 & 2.10 \\
\hline
\end{tabular}

Table 7. Numerical blow-up times, number of iterations and order of the approximations obtained for $q=4$, $p=1$.

\begin{tabular}{|c|c|c|c|}
\hline$I$ & $T_{h}$ & $n$ & $\mathrm{~s}$ \\
\hline 16 & 0.267491 & 12557 & - \\
32 & 0.262877 & 15093 & - \\
64 & 0.261506 & 20507 & 1.75 \\
128 & 0.261106 & 31642 & 1.77 \\
256 & 0.260990 & 54892 & 1.78 \\
512 & 0.260957 & 116438 & 1.81 \\
1024 & 0.260948 & 360972 & 1.87 \\
\hline
\end{tabular}

Table 2. Numerical blow-up times, number of iterations and order of the approximations obtained for $q=3$, $p=2$.

\begin{tabular}{|c|c|c|c|}
\hline$I$ & $T_{h}$ & $n$ & $\mathrm{~s}$ \\
\hline 16 & 0.193186 & 8599 & - \\
32 & 0.189180 & 10386 & - \\
64 & 0.187970 & 14159 & 1.73 \\
128 & 0.187613 & 21902 & 1.76 \\
256 & 0.187509 & 38129 & 1.78 \\
512 & 0.187479 & 83182 & 1.79 \\
1024 & 0.187471 & 260478 & 1.91 \\
\hline
\end{tabular}

Table 4. Numerical blow-up times, number of iterations and order of the approximations obtained for $q=4$, $p=4$.

\begin{tabular}{|c|c|c|c|}
\hline$I$ & $T_{h}$ & $n$ & $\mathrm{~s}$ \\
\hline 16 & 0.194600 & 8702 & - \\
32 & 0.190565 & 10653 & - \\
64 & 0.189347 & 14767 & 1.73 \\
128 & 0.188989 & 23175 & 1.77 \\
256 & 0.188885 & 40621 & 1.78 \\
512 & 0.188855 & 85542 & 1.79 \\
1024 & 0.188847 & 262486 & 1.91 \\
\hline
\end{tabular}

Table 6. Numerical blow-up times, number of iterations and order of the approximations obtained for $q=4$, $p=2$.

In the following, we also give a plot to illustrate our analysis. In the figure below, we can see that the numerical solution blows up in a finite time at the last node. 


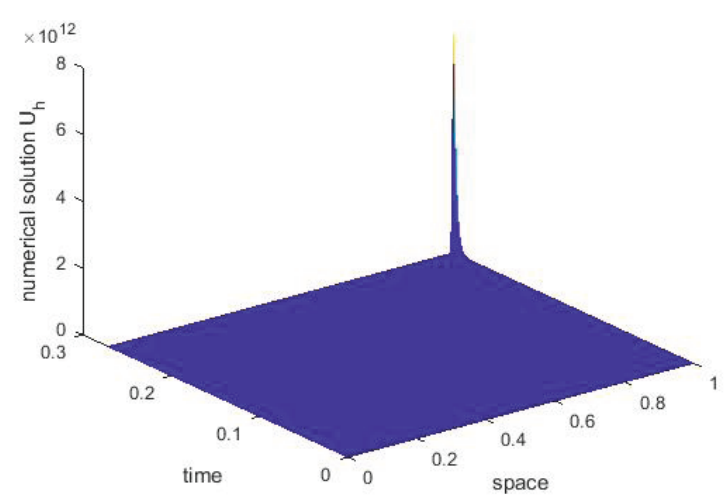

Figure 1. Evolution of the numerical solution for $\mathrm{I}=128, \mathrm{p}=1$ and $\mathrm{q}=3$

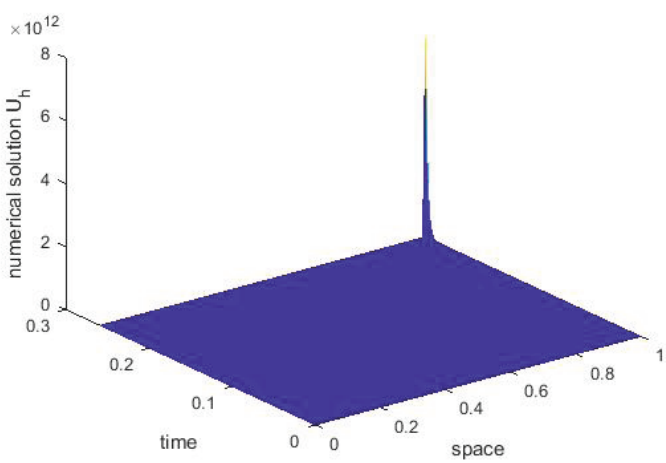

Figure 2. Evolution of the numerical solution for $\mathrm{I}=128, \mathrm{p}=3$ and $\mathrm{q}=3$

\section{References}

Abia, L. M., Lopez-Marcos, J. C., \& Martinez, J. (1996). Blow-up for semidiscretizations of reaction-diffusion equations. Appl. Num. Math, 20, 145-156.

Gomez, J. L., Márquez, V., \& Wolanski, N. (1991). Blow up results and localization of blow up points for the heat equation with a nonlinear boundary condition. Differential Equations, 92, 384-401.

Hairer, E., Nørsett, S. P., \& Wanner, G. (1993). Solving Ordinary Differential Equations I. Nonstiff Problems. Springer Series in Comput. Math. (2nd ed.). Springer-Verlag.

Hirota, C., \& Ozawa, K. (2006). Numerical method of estimating the blow-up time and rate of the solution of ordinary differential equations an application to the blow-up problems of partial differential equations. J. Comput. Appl. Math., 193, 614-637.

Hu, B., \& Yin, H. M. (1994). The profile near blow-up time for solution of the heat equation with a nonlinear boundary condition. Amer. Math. Soc. Transl., 346, 117-134.

Levine, H. A., \& Payne, L. E. (1974). Nonexistence theorems for the heat equation with nonlinear boundary conditions and for the porous medium equation backward in time. Journal of Differential Equations, 16, 319-334.

Ozalp, N., \& Selcusk, B. (2015). Blow-up and quenching for a problem with nonlinear boundary conditions. Electronic Journal of Differential Equations, 1-11.

Taha, M., Toure, K., \& Mensah, E. (2012). Numerical approximation of the blow-up time for a semilinear parabolic equation with nonlinear boundary equation. Far East J. Appl. Math., 60, 125-167.

Toure K. A., N'Guessan K., \& Diabate, N. (2015). Blow-up for semidiscretizations of some reaction-diffusion equations with a nonlinear convection term. Global Journal of Pure and Applied Mathematics, 11, 4273-4296.

Ushijima, T. K. (2000). On the approximation of blow-up time for solutions of nonlinear parabolic equations. Publ. RIMS, Kyoto Univ., 36, 613-640.

Wang, M., \& Wu, Y. (2001). Global existence and blow-up problems for quasilinear parabolic equations with nonlinear boundary conditions. Appl. Math. Comput., 121, 117-134.

Yang, X., \& Zhou, Z. (2016). Blow-up problems for heat equation with nonlinear piecewise neumann boundary condition. Journal of Differential Equations, 261, 2738-2783.

\section{Copyrights}

Copyright for this article is retained by the author(s), with first publication rights granted to the journal.

This is an open-access article distributed under the terms and conditions of the Creative Commons Attribution license (http://creativecommons.org/licenses/by/4.0/). 